On the other hand Heeger argues that the microwave measurements of his group demonstrate that the conductivity peak is real, that the Peierls distortion is not expected to be detectable with the $x$-ray techniques used up to now and that his optical experiments show the existence of a collective mode in $\sigma(\omega)$ at $\omega \approx 0$.

\section{Summary}

It is evident that true high temperature superconductivity in 1-D systems is not possible. The Fröhlich collective mode which is sort of a remnant of superconductivity has been observed in KCP and possibly in TTF TCNQ. Whether fluctuations into the superconducting state around $T_{p}$ really can give huge dc conductivities is still a matter of controversy both experimentally and theoretically. Although many workers in the field got attracted by the idea of high $T_{c}$ superconductivity, other aspects soon turned out to be at least as interesting. In my opinion the real achievement has been to open up a new area of solid state physics by demonstrating that by applying the full spectrum of solid state physics and not just standard materials research techniques, systems of the complexity of KCP and the TCNQ salts can be understood.

REFERENCES
1. H.R. Zeller, Advances in Solid State Physics,
Vol. 13, p. 31. Pergamon, Vieweg (1973).
2. L.B. Coleman, M.J. Cohen, D.J. Sandman,
F.G. Yamagishi, A.F. Garito and A.J. Heeger,
Solid State Commun. 12, 1125 (1973).
3. R.E. Peierls, Quantum Theory of Solids, p. 108.
Clarendon Press, Oxford (1955).
4. H. Fröhlich. Proc. Roy. Soc. A 223, 296 (1954).
5. P.A. Lee, T.M. Rice and P.W. Anderson, Solid
State Commun. 14, 703 (1974).

\title{
The Second Europhysics Conference of the Section of Macromolecular Physics
}

\author{
Sorrento, Italy, 1-3 May 1974
}

Postponed from October 1973 to May 1974, due to the epidemia in Naples, the Conference gathered 140 physicists in an unusually rainy Sorrento.

It was devoted to a highly controversial topic: The state of order in amorphous polymers.

$\mathrm{X}$-ray and electron diffraction patterns, together with some speculations on the dense packing of amorphous polymers compared with the crystalline state, has led to the idea that some local order, characterized by a parallel arrangement of chains in a liquid crystal fashion should exist. Illustrative models like the bundle model or the fringed micelles model have been proposed. For a quantitative approach however, only two extreme and contradictory models are available for comparison with experiments:

- The meander model of Pechhold and Blasenbrei which starts from the crystalline order and introduces well defined defects and superstructures to calculate a thermodynamically stable state compatible with the macroscopic isotropy and with the morphological and dynamic properties of polymer melts.

- The random coil model which extrapolates the gaussian statistics of isolated molecules in solution to the densely packed interpenetrating chains in the solid.

Papers explicitly dealing with the meander model mostly treated the formal development of the model in order to take into account new morphological and dynamic experiments (Pechhold, Hauber, Anthony). Optical transforms of ordered models including the meander model have been shown to give patterns closely resembling the electron diffraction patterns of some amorphous polymers (Bodor).
The most pertinent experimental contributions dealt with techniques appropriate to the direct detection of order at a scale of the order of about $30-50 \AA$.

Neutron scattering experiments on fully deuterated chains dispersed in a matrix of the same protonated polymer have been reported by one French and one German team (Janink, Kirste). They both conclude that not only is the radius of gyration of a chain in the amorphous bulk equal to that in a $\theta$ solvent (a prediction of the random coil model), but also that the scattering envelope fits the gaussian distribution down to $30 \AA$.

The study of density fluctuations by light and X-ray scattering (Wendorff, Ruland), as well as anisotropy fluctuation by anisotropic light scattering (Dettenmaier), above and below the glass transition temperature lead to the conclusion that no "frozen " density fluctuation, as would be expected from regions of local order, is needed to explain intensities and angular dependences which can be well understood in terms of normal thermal fluctuations.

A criticism of the spurious short range peaks introduced by termination errors in the calculation of the radial distribution function by Fourier Transform of the electron diffraction patterns has called the attention to possible misinterpretations (Voigt-Martin).

The conclusion of one paper on NMR line shape analysis of polyethylene melts is the existence of bundles with life times smaller than $10^{-4}$ seconds (Gölz). This result must, however, be treated with caution since an apparent superposition of lorentzian lines can arise from a non-exponential autocorrelation function as produced by a number of possible causes, such as normal entanglements.

If the general impression coming from these experiments is that order does not extend over more than a few angströms, it is fair to add that the "meander school" has claimed that some of this experimental evidence (such as the neutron scattering results) can equally well been explained by the meander model.

Other papers, less directly linked with this controversy, were concerned with :

- The calculation of vibrational modes in chains with well defined types of geometrical or stereochemical defects (Zerbi) and the determination of rotational isomerism in the solid.

- The role of short range order in rubber elasticity.

- The thermodynamics of the amorphous and glassy states incorporating an order parameter.

One session was devoted to the quite different order present in mesomorphic phases of block copolymers. Exciting new possibilities are opened by the ability to obtain monodomain samples. Indirect evidence for disorder in amorphous polymers arises from the lack of intrinsic optical anisotropy of cylindrical microphases $150 \AA$ in diameter (Keller). The existence of spherical microphases in block copolymer cubic microphases should be re-examined in the light of the quite different cubic structures of soaps and lecithines (Luzzati).

If the conference did not succeed in reaching a final conclusion on the state of order in amorphous polymers, the presentation of a series of new experiments and the confrontation of points of view has certainly contributed a step in the direction of a widely accepted model of polymer solid.
G. Weill, Strasbourg 